\title{
Development of microforming process combined with selective chemical vapor deposition
}

\author{
Kazushi Koshimizua ${ }^{a}$, Tetsuhide Shimizu, and Ming Yang \\ Tokyo Metropolitan University, 6-6 Asahigaoka, Hino-shi, Tokyo 191-0065, Japan
}

\begin{abstract}
Microforming has been received much attention in the recent decades due to the wide use of microparts in electronics and medical purpose. For the further functionalization of these micro devices, high functional surface with noble metals and nanomaterials are strongly required in bio- and medical fields, such as bio-sensors. To realize the efficient manufacturing process, which can deform the submillimeter scale bulk structure and can construct the micro to nanometer scale structures in one process, the present study proposes a combined process of microforming for metal foils with a selective chemical vapor deposition (SCVD) on the active surface of work materials. To clarify the availability of this proposed process, the feasibility of SCVD of functional materials to active surface of titanium (Ti) was investigated. CVD of iron (Fe) and carbon nanotubes (CNTs) which construct CNTs on the patterned surface of active Ti and non-active oxidation layer were conducted. Ti thin films on silicon substrate and Fe were used as work materials and functional materials, respectively. CNTs were grown on only Ti surface. Consequently, the selectivity of the active surface of Ti to the synthesis of Fe particles in CVD process was confirmed.
\end{abstract}

\section{Introduction}

Because of low cost, high productivity and stability of process, the demand of micro press forming is increasing to realize small devices made by metallic parts taking advantage of good properties of metals, such as mechanical strengths, electrical conductivity and chemical resistance. Especially for medical devices, biocompatibility of metal has been highly required, and its miniaturization technology is needed. Additionally bio-devices with noble metal or nanomaterials, such as gold (Au), platinum (Pt) and Carbon nanotubes (CNTs) on the surface, are often used in bio-technology by exerting good functions like detecting antibody [1] or catalytic reaction [2]. And, the nano-structuration technologies for functional surface are also required to increase these functions [3]. Therefore, the process for the functional surface is importance in the fabrication of bio- and medical devices.

In the present study, a process with the combination of micro press forming and fabrication of the functional surface for metal foils was proposed. This process can realize the submillimeter structure of metal foils and micro to nanometer structures of functional materials in one step. In this process,

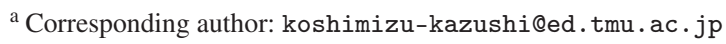

This is an Open Access article distributed under the terms of the Creative Commons Attribution License 4.0, which permits unrestricted use, distribution, and reproduction in any medium, provided the original work is properly cited. 


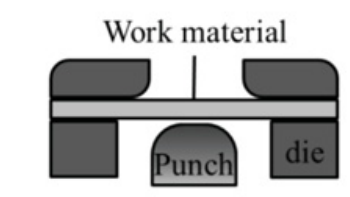

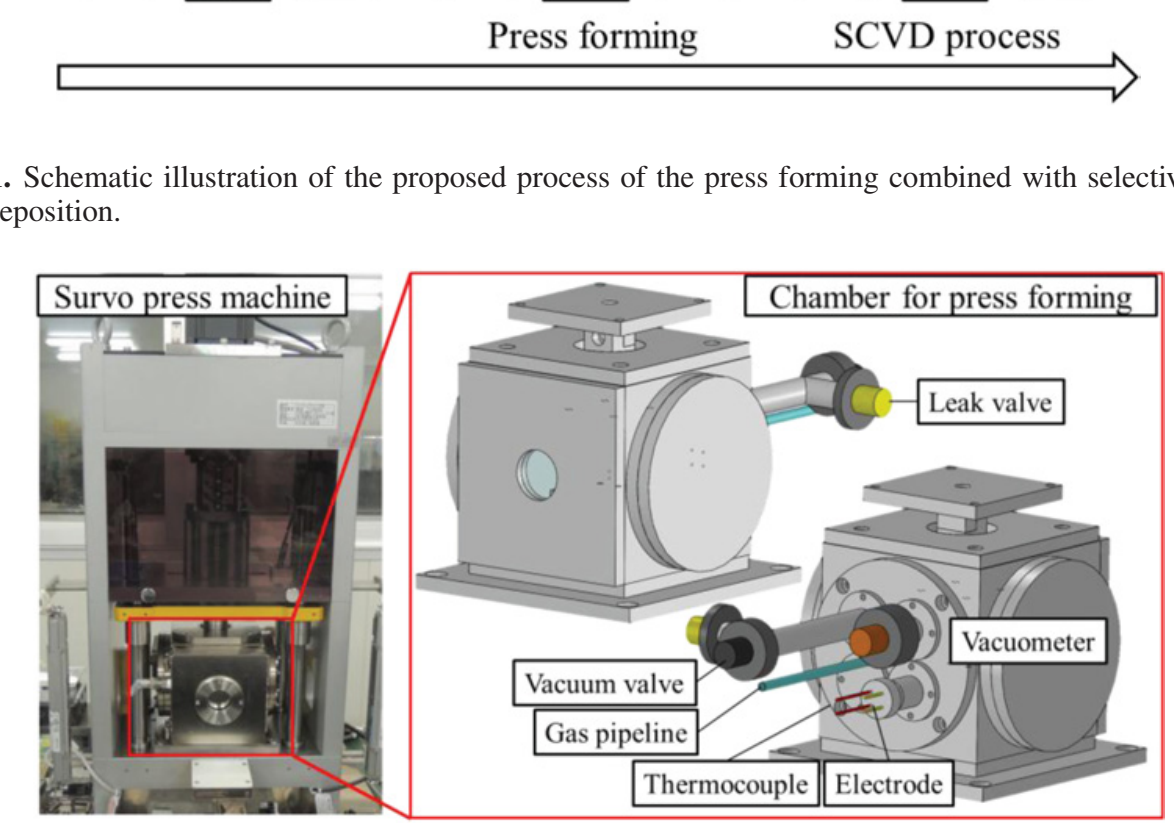

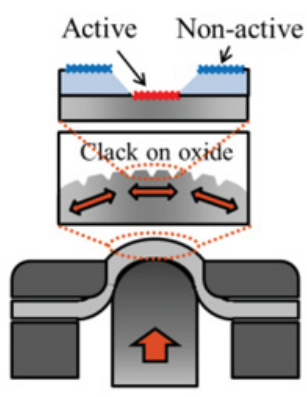

Press forming

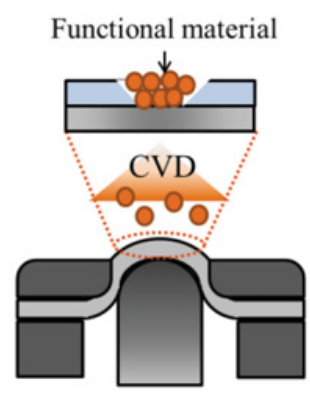

SCVD process

Figure 1. Schematic illustration of the proposed process of the press forming combined with selective chemical vapour deposition.

Figure 2. The appearance and schematic illustration of developed press forming system.

selective chemical vapor deposition (SCVD) for active surface was selected to fabricate the functional surfaces. V. Mohammadi et al. reported that, active surface of Si has better selectivity than non-active surface of $\mathrm{SiO}_{2}$ in metal CVD process. Thus, these metal thin films can be deposited selectivity for oxide and non-oxide patterned surface [4]. As shown in Fig. 1, deformation process may break oxidation layer of metal foil. Thus, active and non-active surface may also patterned on metal foil, and the functional materials can be deposited on only crack area by using SCVD process.

The aim of this study is to clarify the mechanism of crack patterning of oxidation layer and feasibility of the SCVD process for cracks. In this study, the behaviour of strain and thickness of oxidation layer for the cracks and feasibility of SCVD for active and non-active surface were investigated.

\section{Development of system for press forming combined with CVD}

To conduct micro press forming combined with CVD, press forming system with heating of work materials and controlling of atmosphere was developed. As shown in Fig. 2 and Fig. 3, this system mainly consists of a servo press machine, vacuum chamber and die for bulge forming. The die was interlocked with the servo press machine by bellows. There are some equipment such as leak valve, vacuometer, vacuum valve, gas line, thermocouple and electrode in back side of chamber for the 

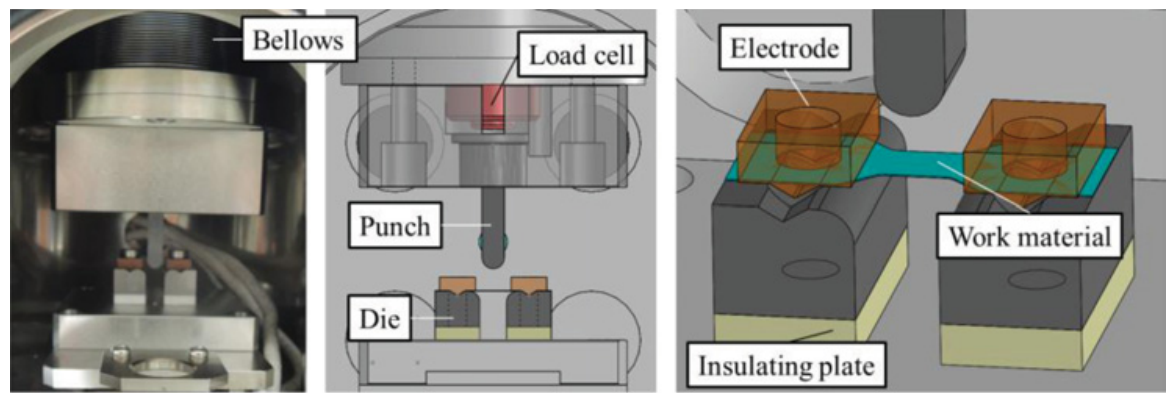

Figure 3. The appearance and schematic illustration of dies for bulging process.

Table 1. Input mechanical properties of specimens and FE simulation conditions.

\begin{tabular}{|c|c|}
\hline Specimen & $\mathrm{Ti}$ \\
\hline Mass density [mg/ $\mu \mathrm{m}]$ & $4.5 \times 10^{-9}$ \\
\hline Young's modulus [GPa] & 110 \\
\hline Yield stress [MPa] & 330 \\
\hline Poisson's ratio & 0.34 \\
\hline Friction coefficient & 0.1 \\
\hline
\end{tabular}

functions of heating and atmosphere controlling. The career gas and precursor for CVD could be introduced from gas line. As shown in Fig. 3, heating of the work materials are achieved by resistance heating using copper electrode.

\section{Feasibility of the cracks of oxidation layer by bulge forming}

\subsection{Finite element analysis of bulge forming and calculation for cracks}

To confirm the effect of the strain of work material and thickness of oxidation layer on the width of the cracks, finite element analysis of bulge forming, calculation for cracks spacing and observation of surface cracks after bulge forming were conducted. In this present study, Ti foil was used as work material.

To investigate the strain of deformed area of $\mathrm{Ti}$ foil, the bulging process was analysed by using ABAQUS ver. 6.13. The schematic illustration of the model is shown in Fig. 4(a). Table 1 shows the mechanical properties of Ti foil and FEM conditions. Where, the mesh size of specimens was $25 \mu \mathrm{m} \times 25 \mu \mathrm{m}$ and the punch stroke was $2 \mathrm{~mm}$ from the punch contacted with Ti foil. As shown in Fig. 4(b), the strain was concentrated around the corner radius of the punch.

B.A. Latella et al. [5] reported that the crack space of oxidation layer can be observed at the area, where the strain was distributed. The crack spacing was considered to expose the active surface of work materials. The crack spacing of oxidation layer is calculated by equation (1) [5],

$$
C s=\sigma_{c} t \tau^{-1}
$$

where, $C s$ is the crack spacing, $\sigma_{c}$ is the fracture strength, $t$ is the thickness of oxidation layer, and $\tau$ is the interfacial shear strength. As shown in Fig. 5, when the thickness of oxidation layer was $3 \mathrm{~nm}$ [6], $100 \mathrm{~nm}$ and $150 \mathrm{~nm}$, the cracks spacing were increased with strain and thickness. 

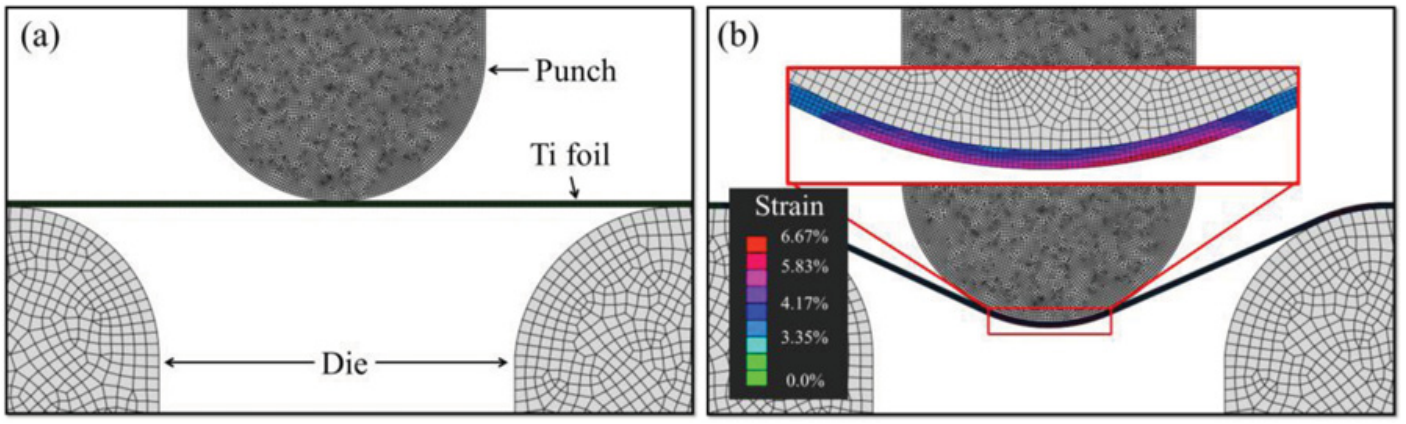

Figure 4. The schematic illustration of FEM (a) model, (b) distribution of strain under $2 \mathrm{~mm}$ punch stroke.

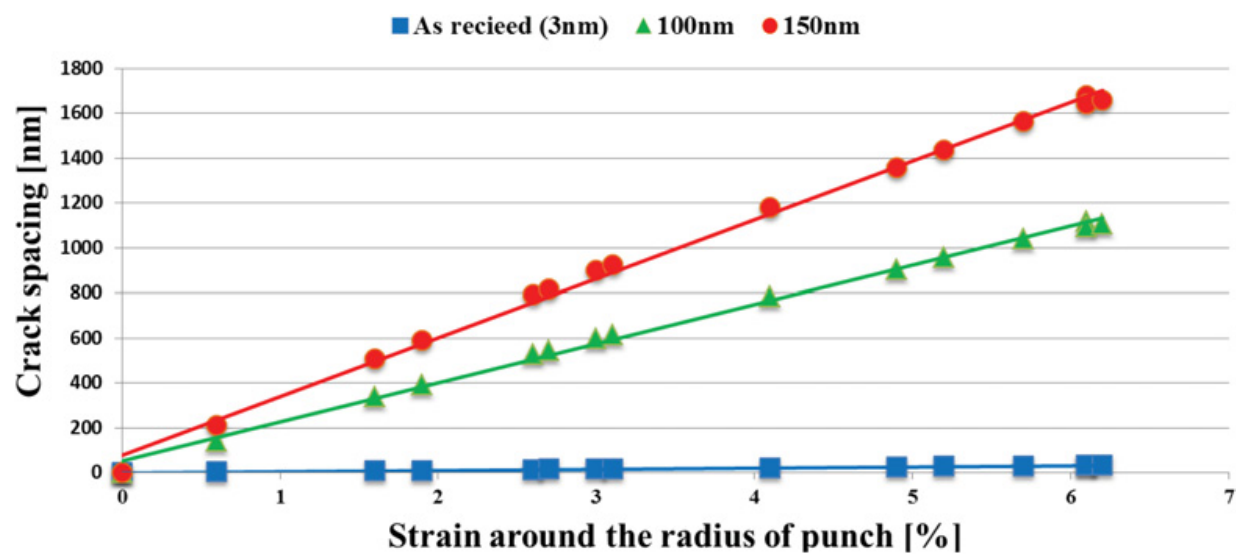

Figure 5. Calculation results of crack spacing of oxidation layer for different strain and thickness.

Table 2. Conditions of bulge forming.

\begin{tabular}{|c|c|}
\hline Punch stroke $[\mathrm{mm}]$ & 2 \\
\hline Strain rate $[\mathrm{mm} / \mathrm{s}]$ & 0.01 \\
\hline Thickness of oxidation layer [nm] & 20 (as received), 100,150 \\
\hline
\end{tabular}

\subsection{Verification of the cracks by practical deformation}

\subsubsection{Experimental}

To confirm the influence of thickness of oxidation layer for crack spacing, Ti foils which had different thickness of oxidation layer were prepared. The thickness of Ti foil was $0.1 \mathrm{~mm}$. These Ti foils were annealed at $700{ }^{\circ} \mathrm{C}$ for 180 and $300 \mathrm{~s}$ to synthesize the oxidation layer with a thickness of 100 and $150 \mathrm{~nm}$, respectively. After the annealing, these Ti foils were deformed by bulge forming system. Those conditions were shown in Table 2.

\subsubsection{Result of surface cracks}

As shown in Fig. 6, the surfaces of Ti foils after the deformation were observed by scanning electron microscope (SEM). As shown in Fig. 6(b) and (c), there were some cracks on the surface of Ti foil. It was considered that these cracks were occurred by the difference of elastic modulus of bulk and oxidation 

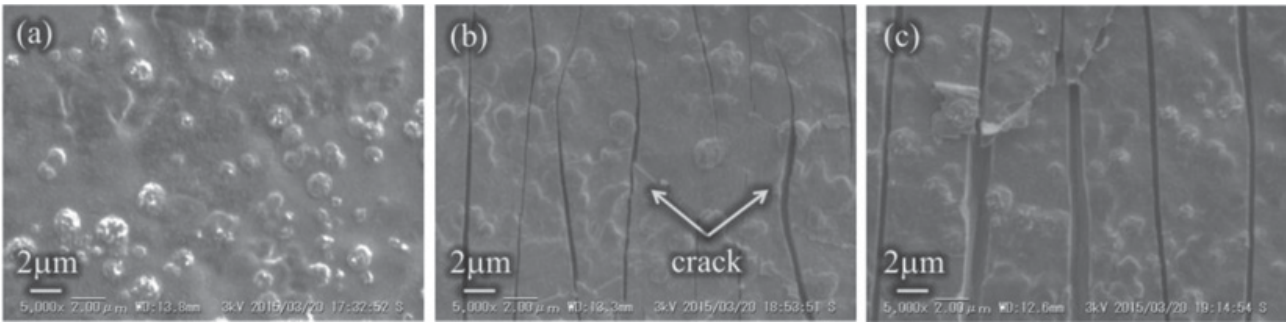

Figure 6. SEM images of deformed Ti foils (a) as received (thickness: $20 \mathrm{~nm}$ ), (b) deformed $6.2 \%$ of strain (thickness: $100 \mathrm{~nm}$ ), (c) deformed $6.2 \%$ of strain (thickness: $150 \mathrm{~nm}$ ).

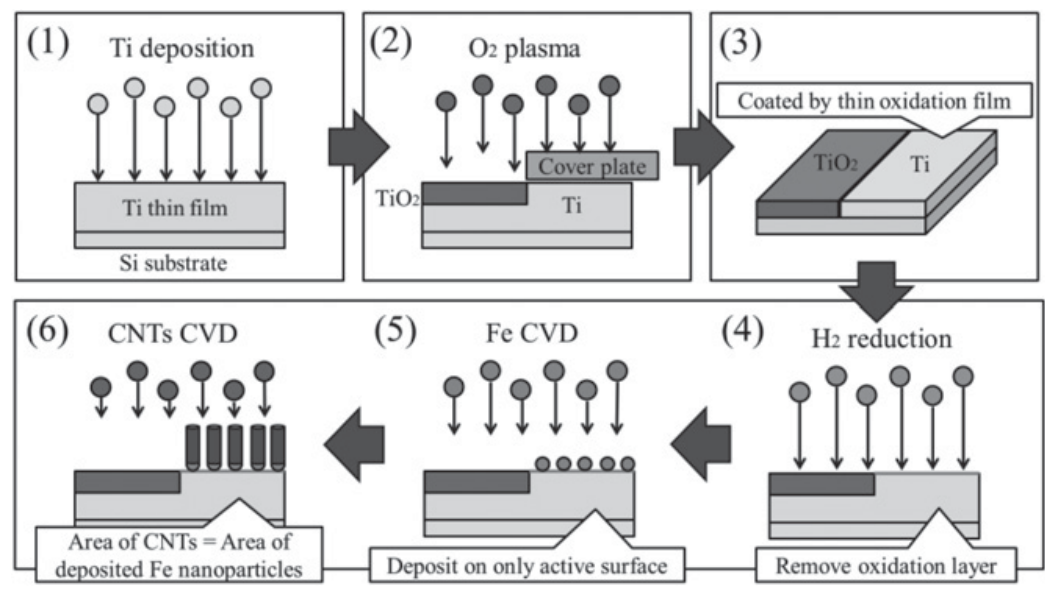

Figure 7. The schematic illustration of experiment to investigate the feasibility of SCVD.

layer of Ti. Additionally, the crack spaces of Fig. 6(c) became larger than (b). Therefore, influence of strain and thickness of oxidation layer for cracks can be confirmed.

\section{Feasibility of SCVD process for active and non-active surface}

\subsection{Experimental}

To investigate the selectivity of CVD process for active and non-active surface, CVD process was conducted to patterned $\mathrm{Ti}$ and $\mathrm{TiO}_{2}$ surface. As shown in Fig. 7, thin Ti films were deposited by electron cyclotron resonance spfuttering machine (ELIONIX Corp.) on Si substrate. The thickness of Ti was $500 \mathrm{~nm}$. $\mathrm{TiO}_{2}$ film was patterned on $\mathrm{Ti}$ film by using oxide plasma machine and the cover plate. After preparing this patterned substrate, hydrogen reduction process was conducted to remove thin oxidation films on Ti surface. Thus, active surfaces of Ti were exposed. In this proposed study, Iron nanoparticles were used as functional materials of CVD process. Fe CVD process was conducted with precursor of ferrocene [Fe(C5H5)2]. Since the Fe particles will be a catalyst for the growth of CNTs, CNTs were synthesized to confirm the deposited location of the Fe nanoparticles on the substrate. 


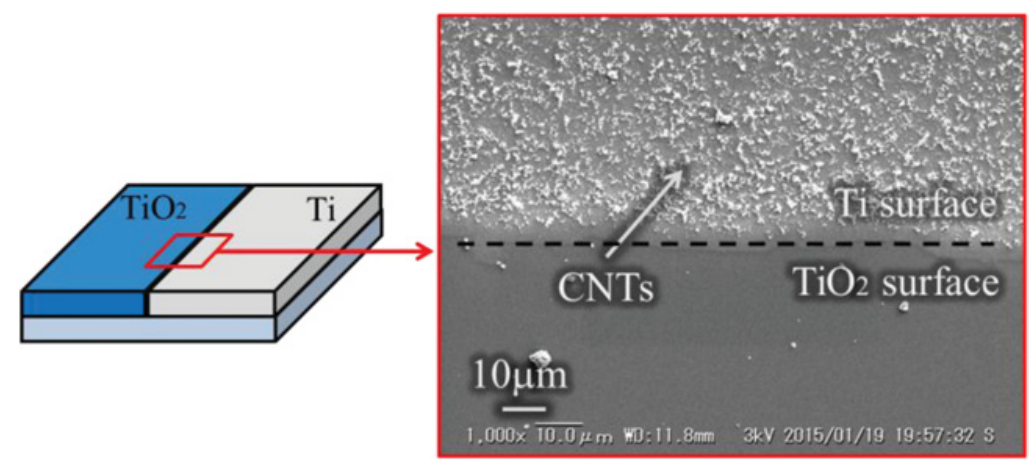

Figure 8. SEM image of $\mathrm{Ti}$ and $\mathrm{TiO}_{2}$ patterned surface after CNTs CVD process.

\subsection{Result of CNTs CVD}

As shown in Fig. 8, the surfaces of Ti thin film after CNTs CVD process were observed by SEM and energy dispersive X-ray analyser (EDX). The CNTs or nano carbon materials were only grown at active surface of Ti. This suggests that Fe nanoparticles were deposited on active surface of Ti better than non-active surface of $\mathrm{TiO}_{2}$. Thus, this result indicated the feasibility of SCVD for active surface of Ti.

\section{Conclusion}

In this study, we proposed the combination process of micro bulging and selective chemical vapour deposition. To conduct this process, the system of press forming combined with CVD process was developed. It has realized press forming with controlling atmosphere and heating work material. In addition, the influences of strain and thickness of oxidation layer of Ti foil were investigated. The cracks of oxidation layer were realized by deformation of Ti foil. The crack space was also increased with thickness of oxidation layer. Finally, to investigate the feasibility of SCVD for active and non-active surface of Ti, Fe CVD process and CNTs CVD process were conducted and CNTs were grown on only active surface of Ti. This suggested that Fe nanoparticles deposited on only active surface in Fe CVD process. Thus, the feasibility of Fe-SCVD process for active surface of Ti was also confirmed.

In the future, the detail of mechanism of cracks and factors to control crack patterns should be investigated, and the feasibility of SCVD process for bulk material should be confirmed for the proposed process.

\section{References}

[1] X. Cao, Y. Ye, S. Liu, Anal. Biochem. 417, 1-16 (2011)

[2] H. Zhao, Y. Wang, Q. Tang, L. Wang, H. Zhang, C. Quan, T. Qi, Int. J. Hydrog.Energy. 39, 9621-9627 (2014)

[3] X. Pang, D. He, S. Luo, Q. Cai, Sens. and Actuators. B 137, 134-138 (2009)

[4] V. Mohammadi, N. Golshani, K.R.C. Mok, W.B. de Boer, J. Derakhshandeh, L.K. Nanver, Microelectron. Eng. 125, 45-50 (2014)

[5] B.A. Latella, B.K. Gan, H. Li, Surf. and Coat. Technol. 201, 6325-6331 (2007)

[6] N.L. Do, E. Garcia-Caurel, N. Bererd, N. Moncoffre, D. Gorse-Pomonti, J. Nucl. Mater. 447, 197-207 (2014) 